(6)

\title{
EINE NEUE WEISHEITSLEHRE?
}

Von WOLFGANG SCHENKEL

Im $\mathcal{F} E A 48$ (1962), 25-35 hat H. Goedicke die Stele des $M n t w-h t p w w$ neu bearbeitet, die seit Griffiths Erstveröffentlichung im PSBA I8 (1896), 195-204 relativ wenig beachtet wurde. Eine Einschränkung ist dabei jedoch angebracht: J. Janssen hat in seiner Traditioneele autobiografie vóór het Nieuwe Rijk (TEA), natürlich nicht im Zusammenhang, fast den gesamten Text neu übersetzt (s. Janssens Register) und einiges zum Verständnis beigetragen. Auch an Goedickes Bearbeitung lassen sich noch verschiedene Korrekturen anbringen. Vor allem aber gibt es Argumente, die gegen die Auffassung von Z. I I ff. als Weisheitslehre sprechen. Doch bevor dieser Hauptpunkt besprochen wird, sollen hier einige Bemerkungen zum ganzen Text und zu den vorausgehenden Zeilen stehen.

I. Zur Datierung. S. 27 (b) wird die Stele, der allgemeinen Ansicht entsprechend, in die I I. Dynastie datiert. Die Argumente, die Goedicke seinerseits dafür anführt, sind nicht stichhaltig.

(a) Der PN Mntw- $h t p w$ ist für eine Datierung ungeeignet, da es $M n \underline{t} w-h \underline{h t p w}$ 's auch in der 12. Dynastie gibt, z. B. heißt so der Besitzer der Stele Kairo 20539, die in der Zeit Sesostris' I. errichtet wurde. Die Namen Fnj-jtjf und Mntw-htpw haben bei Datierungen schon so viel Unheil angerichtet, daß vor solchem Vorgehen nicht genug gewarnt werden kann. Allenfalls hat dieses Verfahren statistischen Wert, und selbst

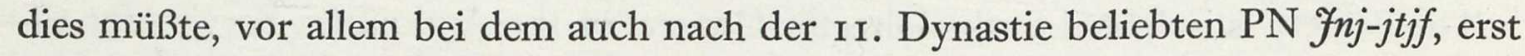
noch auf Stichhaltigkeit überprüft werden.

(b) Stilistisch scheint mir die Stele in die Nachbarschaft von Louvre C. 167 und C. $168 \mathrm{zu}$ passen. ${ }^{\mathrm{I}}$ C. 167 ist in das 25. Jahr Sesostris' I. datiert. Was die Raumaufteilung der Stele betrifft, so muß man sich darüber Rechenschaft geben, daß einmal die I2. Dynastie mehr variiert als die II., und zweitens die Stele aus dem thebanischen Bereich stammt, der in manchen Zügen in dieser Zeit hinter anderen Gegenden nachhinkt. ${ }^{2}$ Sollte man die Anordnung als Archaismus einstufen müssen, so paßte dies sehr schön zu ein paar altertümlichen Zügen in der Orthographie. ${ }^{3}$

(c) Die richtige Datierung, in die 12. Dynastie, ergibt sich, wie ich in FmäS, $\$ 42 \mathrm{~h}$

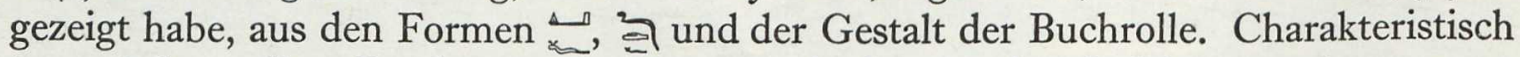
ist auch der endlose Bandwurm von Phrasen. Andere Merkmale führen zu einem ungenaueren, aber immer noch relativ späten Ansatz : das liegende — in der Opferformel ${ }^{4}$ und das Relativpronomen mit Dualstrichen, die offenbar in der Zeit vor der I2. Dynastie

I Fotos bei Moss in Griffith Studies, pl. 47a und 48a.

${ }^{2}$ Vgl. meine Frühmittelägyptischen Studien (FmäS), § 42 i.

3 S. FmäS, § $4 \mathrm{r}$ h.

4 S. FmäS, § 4 . 
nicht gebraucht werden, vgl. I3 A $\beta$ (in $n t j-h n r \cdot j)$; Ny Carlsberg E. 822 B 6; Moskau 4071, 5. Dagegen erscheint all etwa in Siût I 273; 290; 295 und öfter in der Zeit Sesostris' I.

Goedickes Schlüsse, die er S. 29 (k) für die Beziehungen der Ir. Dynastie zu Hermonthis zieht, erledigen sich durch die Datierung. Überhaupt scheinen solche

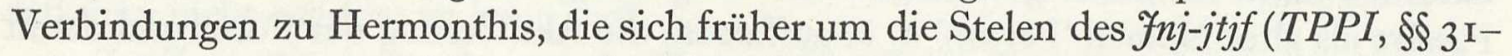
33) rankten, gegenstandslos zu sein. Das in Z. 8 genannte 25. Jahr ${ }^{1}$ kann nur das Ammenemes' I. oder Sesostris' I. sein. Ein späterer Ansatz wird sich nicht empfehlen, die Frage wird jedoch hier nicht weiter untersucht. Eine frühere Datierung dagegen ist praktisch ausgeschlossen, wenn die Stele, wie gezeigt, in die I2. Dynastie gehört. Zwar könnte das geschilderte Ereignis schon lange vor der Abfassung des Stelentextes liegen, doch käme dafür nur das 25. Jahr Mentuhoteps $N b-h p t-R r$ in Frage, das schon mindestens 38 Jahre zurückläge. Eine Bericht über ein so lange vergangenes Ereignis ist wenig wahrscheinlich, zumal Mntw-htpw vermutlich zu dieser Zeit noch nicht in seinen verantwortlichen Ämtern gewesen sein wird, die ihm ja erst Gelegenheit zu seinem segensreichen Wirken gaben.

2. An verschiedenen Stellen kommt das Wort $m r \cdot w t$ 'Liebe' + Suffix vor. In Z. 2 übersetzt Goedicke mißverständlich, wenn nicht falsch übersetzt. Janssen gibt in TEA unter II Bm ro4 dies richtig so wieder: 'wiens heer gemaakt heeft, dat men hem liefheeft'. $m r \cdot w t \cdot f$ heißt mit 'Genitivus objectivus' 'Liebe zu ihm', wie in $Z$. 3 deutlich ist, ${ }^{2}$ und auch die weiteren Belege zeigen werden. Nicht gemeint dagegen kann sein, daß der Herr Mntw$h t p w$ seine Liebe zuwendet, da dies $r \underline{d j} \cdot n n \cdot f n b \cdot f m r \cdot w t \cdot f$ heißen sollte. - Nach S. 29 (r) sei $\$$ in Z. 6 'undoubtedly' mrt wi zu lesen. Diese ungewöhnliche Konstruktion Infinitiv + enklitisches Pronomen, für die man Infinitiv + Suffix in korrektem Ägyptisch erwartete, ist auszuscheiden. Es heißt wieder mit 'Genitivus objectivus' ('Bekannt und

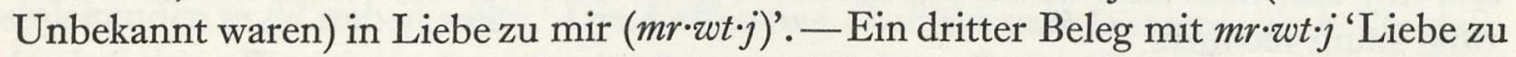
mir' steht dann in Z. Io. Die Konstruktion $m r j \cdot t(w) w j$, die Goedicke S. 3 I (v) zur Wahl stellt, mit pronominalem $t(w)$ 'man' ist im Aä. völlig unmöglich ${ }^{3}$ und auch im frühen Mä. bis in den Anfang der 12. Dynastie nur schwer möglich, und deshalb nur zuzulassen, wenn sich keine andere, einfache Erklärung anbietet. Da über dieses tw 'man' nicht die rechte Klarheit zu bestehen scheint, soll hier die grundsätzliche Entwicklung skizziert werden; die detaillierte Chronologie auszuarbeiten, bin ich hier nicht imstande. ${ }^{4}$

r Vgl. Goedicke, S. 3 r (r).

2 'Die Liebe zu mir war in (diesen und jenen)'; zu $m-\underline{h} t(-n t)=$ 'in' $s$. in meiner noch unveröffentlichten Arbeit über die Grundformen mittelägyptischer Sätze, § I2.

${ }_{4}$ Einer der frühesten Belege für pronominales tw 'man' könnte in doch sind neben $\check{s} d j \cdot t w$ st 'man nimmt sie' auch andere Auffassungen der Stelle möglich, s. James, Hekanakhte, p. 48 (Iо). In Belegen wie Hekanakhte, III 4 und 5 iIr 5 wird man Ellipsen für $\check{s} d j \cdot t w\langle\cdot s\rangle$ und $d d \cdot t w\langle\cdot s\rangle$ ansetzen, s. James, op. cit., p. 48 (7); (10). In dd.tw Ḧekanakhte, II 27 ist natürlich ohne weiteres die folgende Rede als Subjekt anzusetzen. - In jedem Fall wären Belege aus den 'vulgären' Hekanakhte-Briefen noch nicht von Beweiskraft für literarische Texte wie die Mentuhoteps. Sicher nachzuweisen ist pronominales tw 'man' im Sinuhe, s. dazu die in Anm. 2 genannte Arbeit, \$ 29.- Vgl. Westendorf, Passiv, 107-10; Sethe, Verbum, II, $\S \S 190 ; 375$; Gardiner, $E G^{3}, \S 47$ mit Obs. 
Westendorf ${ }^{1}$ glaubt, in dem sich vom MR an herauskristallisierenden $t w$ ' $m a n$ ' unmittelbar das urzeitliche $t j$ 'Person' zu haben, mit dem das $t j / t w$-Passiv gebildet ist. In $s \underline{d} m \cdot t j \cdot f$ träte frühzeitig eine Aktivierung ein. ${ }^{2}$ Die Suffixe bei pronominalem Subjekt wären also bereits früh als Objekte aufgefaßt worden und von da aus natürlich leicht fakultativ geworden. Man mag nun gerne mit Westendorf das $t j$ des Passivs als altes Nomen 'Person' o. ä. auffassen, die Aktivierung der Form und damit die Möglichkeit, tw 'man' herauszulösen, liegt wesentlich später, als Westendorf annahm. Da man bereits im Aä. die - der hypothetischen Entstehung der Suffixkonjugation nach - hybride Bildung w-Passiv + Suffix, wo das Suffix nur als Subjekt fungieren kann, geläufig verwendet, ${ }^{3}$ muß man die Aktivierung der aktiven Suffixbildungen noch ein schönes Stück weiter zurückdatieren, da sie erst die Suffixe als pronominales Subjekt lieferten. Sicher aktivierte Formen des tw-Passivs kann man dagegen erst im MR auftreiben. Der zeitliche Abstand ist also erheblich. Nach so langer Verwendung als Passivelement, und nur als dieses, konnte man kaum noch das alte Nomen 'Person' im Sinn haben.

Nach Westendorfs Ansatz ist es nicht erklärlich, daß man das konstruierbare und in aktivierter Sehweise naheliegende $s \underline{d} m \cdot t j s w$ 'man hört ihn' nicht bildet, statt dessen immer $s \underline{d} m \cdot t j \cdot f$ hat. Die Suffixe hier als Objektspronomina anzusehen, geht nicht an. Dazu gäbe es im Ägyptischen für die ältere, hier allein als Vorbild in Frage kommende Zeit, keine Parallele. Der Verweis auf den Infinitiv ist nicht brauchbar; denn dort fungieren Suffixe genau wie nach einem gewöhnlichen Nomen als 'Zweitgröße', schlechter gesagt als 'Genitiv'. Der Infinitiv hat zwar ein verbales und ein nominales Gesicht, eine Umdeutung dieser Suffixe zu Objekten ist jedoch erst in dem Moment naheliegend, wo die pseudoverbale Konstruktion zum normalen Satztyp wird, wo also Objekte schließlich fast nur noch nach Infinitiven vorkommen können. Für das mä. $s \underline{d} m \cdot t w \cdot f$ darf man also, da keine Vorbilder vorhanden sind, mit einer solchen Umdeutung kaum rechnen.

Für die Entstehung des pronominalen $t w$ 'man' bietet sich eine andere, recht einfache Lösung an. In Sätzen mit tw-Passiv und nominalem Subjekt ( $s \underline{d m} \cdot t w$ hrzv) war es leicht, nach $s \underline{d} m \cdot f$ hrw die passive Konstruktion in eine aktive umzuinterpretieren. Aus 'Die Stimme wird gehört' macht man ein 'Man hört die Stimme'. Äußerlich wird dabei nichts geändert. Aus dieser Konstruktion wurde $t w$ 'man' dann herausgelöst, schließlich sogar zum enklitischen Pronomen ${ }^{4}$ weitergebildet. ${ }^{5}$ Das $t w$-Passiv mit pronominalem Subjekt wurde dabei überhaupt nicht berührt; es lebte als einheitliche Form weiter. Selbst das nun nahe liegende $s \underline{d m} \cdot t w$ sw 'man hört ihn' hat man offenbar - von dem unsicheren šdj.tw S. 7, Anm. 4 abgesehen-erst in der I8. Dynastie gelegentlich gebildet. ${ }^{6}$ Das, wohl im Laufe des MR, neu entstehende $t w$ 'man' hat mit dem alten Formelement $t j$ 'Person' o. ä. nichts als den (lautgesetzlich veränderten) Lautkörper gemein. Die eigenartige Wellenbewegung, die Westendorf, Passiv, 108 bemerken will,

I Passiv, 8I; 108. Im folgenden ist nach $A \ddot{a} G$, $\S$ I 77 für die Formen vor dem Mä. immer $t j$ eingesetzt, was Westendorf noch nicht berücksichtigen konnte.

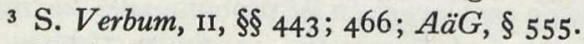

2 Wahrscheinlich zur gleichen Zeit wie beim $s \underline{d m} \cdot f$.

5 Nach Analogie der Pluralsuffixe = enklitischen Pronomina?

6 Etwa jth.tw st 'man schleppt sie', Urk. Iv, 658, 4. 
existiert also nicht. Die von Westendorf in Anm. I zitierte Auflösung synthetischer Formen ist nicht unbedingt ins Spiel zu bringen, zumal die Formeln 'synthetisch' und 'analytisch' derart schemenhaft sind, daß sie für die damit gefaßten Erscheinungen recht wenig besagen.

Für die Stele des $M n t w-h t p w$ ergibt sich aus diesem etwas lang gewordenen Exkurs, daß man nicht $m r j \cdot t(w)$ wj lesen darf.

3. Zu Z. 4 mit Anm. (h) auf S. 28. hm ohne Suffix in der gleichen, 'indirekten' Ausdrucksweise steht in TPPI, §32, 7: '[(Sie) liegen auf] dem Bauch (wört1.: ihren Bäuchen), 0 findet.'

4. Zu Z. 6-7 mit Anm. (o) auf S. 30. Daß (mit in Z. 7 ergänztem $A$ ) ein imperfektisches Partizip sein soll, leuchtet nicht ein. In der Phrase $k r s ~ j z w j u$. ä. wird nie $A$ geschrieben, s. TEA, II, Ga 8; I2-I4. Die Übersetzung der Stelle gibt keinen brauchbaren Zusammenhang: eine Erwähnung aller Elenden $\left(m_{3} j<m_{3} r\right)$ kann nicht an das Bestatten der Greise angehängt werden. Man begräbt zwar die Greise - ganz abgesehen von ihrem Reichtum - , aber den Armen gegenüber rühmt man sich gerne anderer Wohltaten. Falls wirklich $m_{3 j}$ 'arm' dasteht, müßte am Anfang der Z. 7 noch ein ganzes Wort stehen, etwa ${ }_{4}^{\leftarrow}$ : '(Ich bin) ein von den Elenden Geliebter'. Es spricht aber etwas anderes überhaupt gegen die Lesung $m_{3 j}$ 'arm': das Wort könnte man sich zwar so geschrieben denken, aber in Z. Io und I 5 wird es ohne 4 geschrieben: 3 $m_{3 j}$ ' 'arm' ist nun deshalb noch nicht ganz auszuschließen, bleibt aber fraglich, solange man es nicht in einen ansprechenden Kontext stellen kann.

5. Die vermeintliche Weisheitslehre. In Z. I I möchte Goedicke eine 'Lehre' beginnen lassen. Gegen diese Deutung lassen sich entscheidende Gegenargumente geben:

(a) Der unbeholfene Infinitivstil, den die 'Lehre' hätte. Eine derartige Sammlung von abgebrochenen Überschriften kann schlecht eine Rede darstellen, die nach dem einleitenden $m \underline{d} d$ stehen sollte. Die Auffassung Griffiths und Janssens, die beide statt der Infinitive Partizipien lesen, steht dagegen mit dem allgemeinen Sprachgebrauch in Übereinstimmung.

(b) Den Satz $*$ and (is) as follows' zu übersetzen, ist nicht möglich. Hier projiziert Goedicke eine in europäischen Sprachen geläufige Konstruktion ins Ägyptische: das Präpositionalattribut. Wollte man $\star \$$ als $s b 3 \cdot w t$ 'Lehre' nehmen, so könnte der Satz nur heißen: 'Meine Lehre gehört meinen Kindern'. Dann aber wäre das $m \underline{d} d$ nur mit Gewalt unterzubringen. Da das Präpositional- und, was dem sehr nahe steht, das Adverbialattribut im Ägyptischen noch nicht eingehend untersucht sind, folgen hier ein paar vorläufige Bemerkungen.

Präpositional- bzw. Adverbialattribute sind im Mä. weder an allen Stellen im Satz möglich, noch sind sie bei allen Präpositionen und Adverbien geläufig. Konstruktionen wie *jw $k d \cdot n j m j-r-m \check{s} r-m-t 3-r-\underline{d} r \cdot f p r$ 'Der Heerführer im ganzen Land baute ein Haus'I bzw. *jw ḳd·n bik-jm pr 'Der Diener da baute ein Haus' sind m. W. unmöglich.

I In der historisierenden Sprache der Spätzeit werden solche Sätze konstruiert, vgl. Philensis, I, $6=$ Urk. II, $205,3 \mathrm{f}$.

C 2298 
Die Attribute können nicht vor dem Objekt im Satz mit trans. Verbum stehen; oder, anders gesagt, sie stehen nur dort, wo die ihnen formal völlig gleichen präpositionalen bzw. adverbialen Ausdrücke stehen, die sich auf den ganzen Satz beziehen. Das Ägyptische scheint hier die verschiedenen Satzebenen, die in europäischen Sprachen klar getrennt sind, nicht ganz so deutlich abzusondern. Daß das Ägyptische den Unterschied kennt, zeigt sich z. B. dann, wenn am Satzende sowohl ein Präpositionalattribut als auch eine weitere präpositionale Bestimmung unterzubringen sind: das Attribut folgt dann seinem Bezugswort unmittelbar. ${ }^{I}$ Wie bisweilen die adverbialen Satzteile können Präpositionalattribute zwischen Nomen und indirekten 'Genitiv' eingeschoben werden. ${ }^{2}$ Vielfach stehen statt der Präpositionalattribute europäischer Sprachen andere Konstruktionen, ${ }^{3}$ vor allem Nisben, ${ }^{4}$ die Genitivpartikel + präpositionales Gebilde ${ }^{5}$ und Relativsätze. ${ }^{6}$ Wirklich geläufig sind mit Adverbien nur wenige stehende Wendungen, wie $b 3 k j m,{ }^{7}$ wrjw jm $n b ;^{8}$ mit Präpositionen $N b-r-d r r$ 'Allherr', 8 $r \underline{d} r(\cdot f),{ }^{9} m j k d(\cdot f),{ }^{9} r \quad r w(\cdot f)^{10}$ und Verbindungen mit der Präposition $m$. Das zuletzt genannte $m$ hat wahrscheinlich noch den freiesten Gebrauch; doch ist auch dies stark auf Titulaturen und Formeln eingeschränkt, die entweder gar nicht in einem Satz stehen oder in Aufzählungen nur locker damit verbunden sind. ${ }^{\text {II }}$ Eine freiere Verbindung ist etwa:

\section{$m w$ m jtr.w, swr $t w \cdot f, m r j \cdot k$ \\ $\underline{t} s w m p \cdot t, h n m \cdot t w \cdot f, \underline{d} d \cdot k$}

'Das Wasser in den Flüssen trinkt man, wenn du es willst; die Luft am Himmel atmet man, wenn du es sagst'. ${ }^{12}$ Hier steht Nomen + Präpositionalattribut aber auch nicht völlig im Satzverband: der ganze Ausdruck ist antizipiert.

Gewiß wird eine eingehendere Untersuchung des Präpositional- und Adverbialattributs besser erkennen lassen, was das Ägyptische mit diesen Mitteln zu leisten vermag. Doch wird an der Tatsache, daß die Konstruktion gegenüber europäischen Sprachen stark eingeschränkt ist, nichts zu ändern sein. Für die Stele des $M n t \underline{t} w-h t p w$ ist bereits so viel sicher, daß das von Goedicke vermutete Adverbialattribut äußerst unwahrscheinlich ist. Eine 'Lehre für seine Kinder' kann ägyptisch z. B. sbs:jt jrj·tn·f $h r m s \cdot w \cdot f$, wörtl. etwa eine 'Lehre, die er vor seinen Kindern machte' heißen, wie Kairo $2053^{8}, 8 \mathrm{f}$. zu lesen steht.

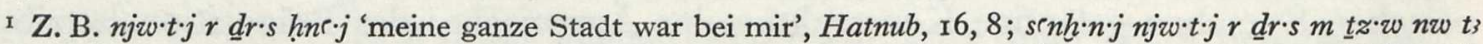
'Ich versorgte meine ganze Stadt in den "Sandbänken" (d. h. im Hungerjahr) mit Lebensmitteln', Hatnub, 24, 9 f.; jw $\zeta m\ulcorner w r d \underline{d} \cdot f m w t \cdot w n n h k r r$ 'Während das ganze Land Hungers starb', Mo'alla, Iv, I5.

2 mšr $r \underline{d} r f n_{j}$ Gbtjw 'die ganze Garnison von Koptos', Koptos, 8, 2; $n n r$ sw $n j$ rnp $\cdot w t$ 'diese Jahre alle', Adm. I3, 2.

${ }_{3} E G^{3}, \S 158 ;$ Lefebvre, $G E C, \S 488$.

$4 h k_{3} \cdot w$ h hrj·w-tp ďs r $t$ ' 'die Häuptlinge in der Wüste', TPPI, § $20 \mathrm{~A} 6$.

5 ḩz'wt'j nt hrr njswt 'meine Gunst beim König', El Bersheh, I, pl. xiv 8.

${ }^{6}[t] w t \cdot w \cdot k$ ntjw $m$ hw'wt-ntr $n t$ S Smcw 'deine Statuen in den Tempeln Oberägyptens', Urk. 1, 305, 10-1 r.

$7 E G^{3}, \S 206$; Borchardt, $Z \ddot{A} S 27$ (1889), 122-4; Sethe, $Z \ddot{A} S 30$ (1892), $126 \mathrm{f}$.

$8 E G^{3}, \S 206$. ' $\quad E G^{3}, \S \S 100 ; 158$; Lefebvre, $G E C$, §§ $189 ; 488$. 10 $E G^{3}, \S 100 ; G E C, \S \S 189 ; 488$.

Ir jmj-r mšr $m t_{3} r \underline{d} r \cdot f$ 'der Truppenführer im ganzen Land', Ny Carlsberg E. 822 A 2-3; B $3 ; s r m-h s^{\prime} \cdot t r h \cdot j t$ 'Beamter an der Spitze der $r h \cdot j t$-Menschen', Kairo 20538 , I c I f.; $d b h \cdot t h t p \cdot w ~ m p r W s j r$ 'der Opferbedarf aus

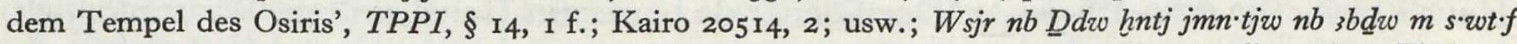
$n b \cdot(w) t$ 'Osiris, der Herr von Busiris, der Erste der Westlichen, der Herr von Abydos, an allen seinen Plätzen', passim.

${ }_{12} \operatorname{Sin} . \mathrm{B} 233 \mathrm{f}$. 
Das Wort, das Goedicke dazu verleitet hat, eine Weisheitslehre anzunehmen, ist * 1 , das nicht im Wb. steht. Janssen übersetzt unter II, Dr I richtig 'onderrichter (der kinderen)'. Neben dem bekannten $s b 3 \cdot w$ 'Lehrer, Erzieher' $W b$. IV, 85 , I ff. gibt es offensichtlich ein $s b 3 \cdot w t j$. Nomina auf $-w$ neben einer von einem Abstraktum weitergebildeten Nisbe sind nicht ganz selten. So steht $3 \underline{t}(j) \cdot w$ 'Erzieher' neben $3 t(j) \cdot w t j$, ${ }^{\text {I }}$ $h m w \cdot w$ 'Technit'2 neben $h m w \cdot w t j,^{3} w t(j) \cdot w$ 'ältester Sohn'4 neben $w t \cdot w t j .5$ S. weiter Fecht, Wortakzent, § I 72 .

Den Satz, den Goedicke als Einleitung einer Lehre sehen wollte, kann man nach allem nur (mit Janssen) übersetzen: 'Ein Erzieher der Kinder durch ruhiges Sprechen', wobei $h r \cdot t$ in üblicher Beschreibung Adverb wäre. ${ }^{6}$

Es folgen nun Einzelbemerkungen zu Z. I I ff.

6. Zu Z. I I f. und Anm. (z) auf S. 33. Goedicke liest $r$ sk·wtj·fj bzw. $d d \cdot t j \cdot f j$. Diese Konstruktion würde dem Sinn der Stelle weniger gerecht als die in verwandtem Kontext geläufige mit $r+s \underline{d} m \cdot t \cdot f \cdot{ }^{7}$ Es geht sicher nicht nur darum, daß der Bittsteller vor seinem Bericht gut behandelt wird, sondern auch während seines Berichts. Nur wenn der Bittsteller bis zum Ende angehört wird, ist sein Zweck erreicht. Was Goedicke vermutlich auf das $s \underline{d} m \cdot t j \cdot f j$ gebracht hat, ist das Suffix $-f$, das sich an nichts Vorausgehendes anlehnt. Für das Ägyptische liegt aber um so weniger ein Mangel vor, als es sich bei den Sätzen mit $r s \underline{d} m \cdot t \cdot f$ um stehende Wendungen handelt, bei denen ohnehin bekannt war, was mit dem Suffix gemeint ist.

7. Z. 13 und Anm. (ag) auf S. 33. Uneretern erhebliches Kopfzerbrechen. Griffith übersetzt: '(It was a kindly (or "beloved") hand), it was a unique complexion (species?)'. Grapow denkt, ${ }^{8} M n t w-h t p w$ sei 'von einer Haut zu den Leuten', d. h. ein Mann von gleichmäßig freundlicher Art. Janssen gibt unter V 24: 'De menschen zijn één huid'. Vielleicht sollte entsprechend der Konstruktion, die Janssen vorschlägt, zu übersetzen sein: '(Eine freundliche Hand ist das, was geliebt wird), die Menschen sind (vor mir) von einer einzigen Art'. Das hieße: Mntw- htpw ist gegen alle ohne Ausnahme zuvorkommend, gegen die Niederen, von denen die Rede war, und gegen die Hohen, auf die er gleich zu sprechen kommt.

8. Zum Schluß folgen die Zeilen II-I6, die vermeintliche Weisheitslehre, in neuer Übersetzung:

(II) ..... (Ich bin) ein Lehrer der Kinder in ruhigem Reden; ein Geduldiger; einer, der nicht mit einem Geringen streitet: (denn) es gibt keinen hochmütigen (und doch) geliebten Vorgesetzten; einer, der freundlich ist, bis er (der Bittsteller) seine Schwierigkeiten gesagt hat, (12) bis er sein Herz ausgeschüttet hat; der seinen Fall anhört, der sein Leid vertreibt; der einen Mann nach Gebühr (?) bescheidet (?); ${ }^{9}$ der $\langle\text { frei }\rangle^{10}$ von Übertreibung ist; der schweigt, wenn man seine

1 Vgl. $A \ddot{a} G, \S 227$.

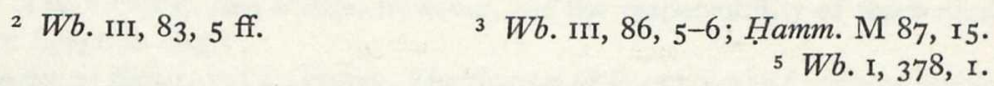

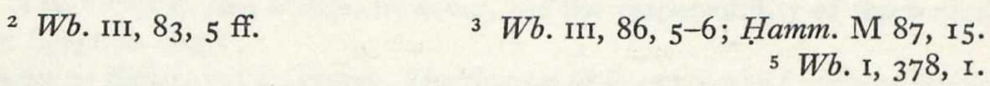

$4 \mathrm{~Wb}$. I, 377, 21 .

6 Vermutlich handelt sich um das absolut gebrauchte feminine Partizip.

7 Vgl. im gleichen Text Z. 14; Ptahhotep, 266; 267.

${ }^{8}$ Bildliche Ausdrücke, 107.

9 Vgl. Kairo 20539, I b $8=T E A$, II, Bm 57; dazu TEA, Text, Ir6.

to $m h_{3} w$ wäre nur mit Verrenkungen in den Kontext einzupassen. Janssen hat es deshalb in seiner Sammlung einfach übergangen. Ein vernünftiger Sinn ergibt sich, wenn man $\beta$ davor einfügt, vgl. die Phrasen $T E A$, vi, J 25; 31 ; Schiffbr. 12 f. 
Gedanken (vor ihm) verbirgt (?); der (I3) gegen jedermann zuvorkommend ist; der sein Gesicht nicht vor dem Hungernden abwendet. Eine freundliche Hand ist das, was geliebt wird. Die Menschen sind (vor mir) von einer Art. Es gibt (bei mir) keine Auflehnung gegen einen Beauftragten (I4) oder irgendeinen Beamten der Verwaltung(?), vielmehr ${ }^{1}$ sagte ich:2 'Neige dein Herz; sei nicht voreingenommen gegen einen Bittsteller, bis er gesagt hat, weswegen er gekommen ist.' Mir wurden die Belange ${ }^{3}$ des Geringen, (15) der Witwen und Waisen in gleicher Weise ${ }^{3}$ berichtet. Ich wirkte [für] sie [alle] (?), ${ }^{4}$ um den, der ins Elend gekommen war, atmen zu lassen. Der gute Charakter eines Mannes taugt ihm mehr als 1000 Gaben in Taten. Das Zeugnis der Menschen liegt (I6) in jenem Ausspruch im Munde der Geringen:5 'Das gute Wesen eines Mannes ist sein Denkmal. Der Charakterlose wird vergessen.' Wenn es nach dem Sprichwort geht, so wird mein guter Ruf in meiner Stadt dauern, und mein Denkmal wird niemals untergehen.

I wpw-hr + Inf. ist im Deutschen wörtlich nicht nachzubilden.

2 Doch wohl nicht zu den Beamten - das wäre Besserwisserei-, sondern als Maxime zu sich selbst.

3 Lies $h r \cdot t$ und $r m j t \cdot t$, wie Griffith hat. Ich sehe hier keinen Grund, von Griffith abzuweichen, der ja bei der ersten Abschrift offensichtlich den Text eindeutig sah. Die grammatischen Überlegungen, die Goedicke in Anm. (ak) und Fußnote 2 auf S. 34 bringt, scheinen mir für die Praxis der Stelentexte nicht ganz zutreffend zu sein.

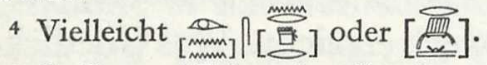

5 S. Gunn, $\mathscr{F} E A$ 12 (1926), 282 mit Fußnote 2. 